\title{
Perfil microbiológico de las infecciones Nosocomiales en el Hospital Universitario Hernando Moncaleano Perdomo de Neiva
}

\author{
Microbiological Profile of Nosocomial Infections at \\ University Hospital Hernando Moncaleano Perdomo
}

\author{
Ivan D Medina; ;onathan Díaz; Giovanni Caviedes Pérez² \\ 1 Semillero Pre ciencia, Programa de Pregrado en Medicina. Universidad Surcolombiana \\ 2 Médico Internista, Farmacólogo Clínico, Epidemiólogo. Docente, Universidad Surcolombiana. \\ Correspondencia: Giovanni Caviedes Pérez, Correo electrónico: giocape@hotmail.com
}

Fecha de recepción: Octubre 08-2013

Fecha de aceptación: Diciembre 17-2013

Medina I, Díaz J, Caviedes G. Microbiological Profile of Nosocomial Infections at University Hospital Hernando Moncaleano Perdomo. RFS - Revista Facultad de Salud. 2013; 5 (2): 41-51

\section{Resumen}

Las infecciones nosocomiales son una de las principales causas de morbimortalidad a nivel hospitalario, el manejo antibiótico constituye el pilar principal para el tratamiento de este tipo de infecciones por lo cual se debe realizar de manera adecuada con el fin de evitar el aumento de la resistencia microbiológica a los antibióticos.

Se determinó el perfil de resistencia microbiológico de las infecciones nosocomiales a través de las MIC en el Hospital Universitario Hernando Moncaleano Perdomo. Se realizó un estudio de tipo descriptivo serie de casos, con un enfoque retrospectivo, en el cual se desarrolló un análisis cuantitativo del perfil de resistencia microbiológica teniendo en cuenta las concentraciones mínimas inhibitorias farmacológicas, mediante la investigación de las bases de datos del departamento de epidemiología y del laboratorio clínico del mismo hospital en el periodo comprendido entre febrero de 2011 y febrero de 2013.

Se analizaron un total de 646 reportes de antibiogramas encontrándose que la mayor cantidad de infecciones intrahospitalarias es debida a gérmenes Gram negativos, destacándose Klebsiella, Pseudomona y E. coli; de los gérmenes Gram positivos el Estafilococo, y de las infecciones por hongos Cándida. La infección nosocomial más prevalente correspondió a las bacteriemias con el 30,7\%, principalmente en la UCl Adultos, seguido de Medicina Interna y de Cirugía. La infección del tracto urinario (ITU) Nosocomial es la segunda causa de infecciones intrahospitalarias con un porcentaje de 19,8\% del total de casos en los servicios de Medicina Interna y UCl adultos.

Palabras Clave: Infección Nosocomial, Concentración Mínima Inhibitoria, Antibiograma, Sensibilidad, Resistencia.

\section{Abstract}

Nosocomial infections are one of the main causes of morbimortality at hospital level. Antibiotic management is the major mainstay for treating these kinds of infections; thereby it should be adequately administered in order to avoid an increase in microbiological resistance to antibiotics. 
It was determined the microbiological resistance profile of nosocomial infections through the MIC at Hospital Universitario Hernando Moncaleano Perdomo. A retrospective case series descriptive study was conducted in which it was developed a quantitative analysis of microbiological resistance taking into consideration the minimum inhibitory pharmacological concentrations. It was searched databases of the hospital's department of epidemiology and clinical lab from february of 2011 to february of 2013.

A total of 646 antibiogram reports were analyzed and it was found that most nosocomial infections are due to negative Gram germs, being Klebsiella, Pseudomona and E. coli, the outstanding ones; of the positive Gram germs Staphylococcus, and of the infections by Candida fungi. The most common nosocomial infection corresponded to bacteremia by $30,7 \%$, main in adult ICU, followed by internal medicine and surgery. Nosocomial UTI is the second cause of nosocomial infections by $19 \%$ of total cases at internal medicine service and adult ICU.

Keywords: nosocomial infection, minimum inhibitory concentration, antibiogram, sensitivity, resistance.

\section{Introducción}

Las infecciones nosocomiales constituyen una de las principales causas de morbimortalidad en las instituciones prestadoras de servicios de salud; el tratamiento antibiótico constituye la piedra angular en el manejo de estas infecciones y de ahí radica la importancia de conocer los porcentajes de sensibilidad y resistencia antibiótica según el perfil epidemiológico de cada institución; dichos perfiles van cambiando con el tiempo por diversos factores tales como: mutaciones en los sistemas de resistencia microbiológica, el uso inadecuado de la terapia antibiótica empírica, el uso rutinario e indiscriminado de antibióticos sin tener en cuenta los porcentajes de sensibilidad y resistencia de la institución, entre otros ${ }^{(1,2)}$.

El Hospital Universitario Hernando Moncaleano Perdomo (HUHMP) es uno de los más importantes centros de referencia de atención en salud en el sur del país, por tanto la importancia de determinar el perfil de resistencia microbiológica con el fin de optimizar los resultados de la terapia antibiótica, la cual se debe realizar de la mejor manera posible utilizando el antibiótico más idóneo según los perfiles de sensibilidad y resistencia para los distintos gérmenes del entorno hospitalario, optimizando así la eficacia del antibiótico para el cual es sensible el microorganismo al usarlo en la mínima concentración inhibitoria (MIC), menguando así los efectos secundarios para el paciente y a su vez evitando el desarrollo de la resistencia microbiana.
El objetivo del presente trabajo fue determinar el perfil de resistencia microbiológico individual de las infecciones nosocomiales a través de las MIC farmacológicas en el HUHMP durante el periodo epidemiológico de febrero 2011 a febrero de 2013 tras la adopción de las guías de manejo antibiótico en el HUHMP.

\section{Materiales y métodos}

El presente estudio es de tipo descriptivo serie de casos, con un enfoque retrospectivo, en el cual se realizó un análisis cuantitativo del perfil de resistencia microbiológica teniendo en cuenta las concentraciones mínimas inhibitorias farmacológicas del HUHMP mediante la investigación de las bases de datos del departamento de epidemiología y del laboratorio clínico del mismo hospital en el periodo que comprende de 2011 hasta el 2013; con el fin de actualizar la situación actual del perfil y determinar si se está brindando un manejo antibiótico adecuado y acorde a las datos establecidos.

Se incluyeron hombres y mujeres de cualquier grupo etario hospitalizados en el HUHMP durante el periodo epidemiológico comprendido de febrero de 2011 a febrero de 2013 de los diferentes servicios y/o unidades de atención con diagnóstico de infección nosocomial a los cuales se les realizó un cultivo con su respectivo antibiograma. Fueron seleccionados los registros de antibiograma de aquellas historias clínicas de pacientes con diagnóstico confirmado de infección nosocomial durante el periodo de estudio. 
La base de datos inicial aportada por el departamento de epidemiología e infectología tomó un total de 819 pacientes que se encontraron hospitalizados con diagnóstico de infección nosocomial, de los cuales se excluyeron 183 pacientes por no cumplir con los requisitos previstos para la investigación; teniendo un total de 636 pacientes aptos para el estudio que se procedieron a revisar. 35 de ellos no reportaron cultivo en la base de datos del HUHMP y por tanto fueron descartados, obteniendo así un total de 601 pacientes con un conteo de 646 antibiogramas.

Se utilizó una ficha de recolección de datos diseñada a partir de los principales criterios a evaluar, tales como: datos de identificación hospitalaria (número de historia clínica, edad del paciente, género, servicio tratante, año y mes en el cual presentó la infección nosocomial) y datos de antibiograma (tipo de infección nosocomial que presentó durante su estancia hospitalaria, agente causal de dicha infección, perfil de sensibilidad y resistencia antibiótica, y concentración mínima inhibitoria (MIC) para cada antibiótico procesado). Los resultados obtenidos fueron registrados en Microsoft Office Excel 2010 mediante el diseño de una ficha habilitada con la extensión de macros en el lenguaje de visual basic diseñada exclusivamente para tal fin.

Se solicitó la aprobación al comité de ética médica de la Universidad Surcolombiana y a la oficina de investigaciones del Hospital Universitario de Neiva para la realización del estudio y el manejo de la información.

\section{Resultados}

\section{Distribución etárea y por género}

Con respecto a la distribución por género de los pacientes encontrados para el trabajo hallamos un total de 197 mujeres correspondientes al $32,78 \%$ y un total de 404 hombres correspondientes a un $67,22 \%$. Para efectos prácticos fraccionamos la población en 3 grupos etareos repartidos (Tabla 1). Para ambos géneros se presentaron picos durante las edades de $\geq 16$ años y $\leq 64$ años con porcentajes de $75 \%$ y $54 \%$ para hombres y mujeres, respectivamente.

Tabla 1. Distribucion según el género y la edad.

\begin{tabular}{lc}
\hline \multicolumn{1}{c}{ Variables } & Resultados \\
\hline Género & n (\%) \\
\hline Hombres & $385(67,4)$ \\
Mujeres & $186(32,6)$ \\
\hline Edad & $\mathbf{n}(\%)$ \\
\hline Menor de 16 años & $40(7)$ \\
16 a 64 años & $390(68,3)$ \\
mayor a 64 años & $141(24,69)$ \\
\hline
\end{tabular}

\section{Distribución de gérmenes e infecciones noso- comiales}

Los principales gérmenes encontrados en el medio intrahospitalario se ilustran en la Tabla 2, de los cuales se tomaron como de mayor importancia los 5 primeros por su impacto en el número de casos presentados asociados a infecciones nosocomiales.

Tabla 2. Distribucion porcentual de gérmenes causantes de infecciones nosocomiales en el HUHMP.

\begin{tabular}{lc}
\hline Germen & N (\%) \\
Klebsiella sp & $168(26)$ \\
Pseudomonas sp & $113(17,49)$ \\
E coli & $96(14,86)$ \\
Estafilococcus sp & $76(11,76)$ \\
Acinetobacte sp & $56(8,67)$ \\
Enterobacter sp & $48(7,43)$ \\
Cándida & $39(6,09)$ \\
Proteus sp & $21(3,25)$ \\
Enterococus sp & $13(2,01)$ \\
Otros & $16(2,47)$ \\
\hline
\end{tabular}

De los 646 casos reportados el mayor porcentaje correspondió a las bacteriemias con el 30,7\%, valor que primó en la Unidad de Cuidados Intensivos (UCI) Adultos, seguido de Medicina Interna y de Cirugía (Tabla 3). 
Tabla 3. Distribución porcentual de infecciones nosocomiales por servicio de atención.

\begin{tabular}{|c|c|c|c|c|c|c|c|c|c|c|c|c|c|c|c|c|c|}
\hline & \multicolumn{2}{|c|}{ Cirugía } & \multicolumn{2}{|c|}{$\begin{array}{c}\text { Gineco- } \\
\text { logía }\end{array}$} & \multicolumn{2}{|c|}{$\begin{array}{c}\text { Infectología } \\
\text { Adultos }\end{array}$} & \multicolumn{2}{|c|}{$\begin{array}{c}\text { Infectología } \\
\text { Pediatría }\end{array}$} & \multicolumn{2}{|c|}{$\begin{array}{l}\text { Medicina } \\
\text { Interna }\end{array}$} & \multicolumn{2}{|c|}{$\begin{array}{l}\text { Neuro } \\
\text { cirugía }\end{array}$} & \multicolumn{2}{|c|}{$\begin{array}{c}\text { Oncohe- } \\
\text { matología }\end{array}$} & $\begin{array}{c}\text { Ortope- } \\
\text { dia }\end{array}$ & \multicolumn{2}{|c|}{$\begin{array}{c}\text { Transplan- } \\
\text { te Renal }\end{array}$} \\
\hline & № & $\%$ & № & $\%$ & № & $\%$ & № & $\%$ & № & $\%$ & № & $\%$ & № & $\%$ & № & № & $\%$ \\
\hline Bacteriemia & 24 & 18,5 & 4 & 26,7 & 2 & 20 & 3 & 75 & 26 & 22,4 & 4 & 9,5 & & & 2 & 5 & 38,5 \\
\hline Ivun & 17 & 13,1 & 4 & 26,7 & & & & & 46 & 39,7 & 8 & 19,0 & & & 4 & 1,1 & 15,4 \\
\hline $\mathrm{NN}$ & 5 & 3,8 & 3 & 20,0 & 2 & 20 & & & 19 & 16,4 & 5 & 11,9 & 2 & 66,7 & 1 & 8 & \\
\hline B.A.C. & 23 & 17,7 & & & 2 & 20 & 1 & 25 & 14 & 12,1 & 1 & 2,4 & & & & 6 & 46,2 \\
\hline Iso Cx Gral & 52 & 40 & & & & & & & & & & & & & & & \\
\hline Iso Ortopedia & & & & & & & & & & & & & & & 2980 &, 6 & \\
\hline Iso Neurocx & & & & & & & & & & & 194 & 45,2 & & & & & \\
\hline $\begin{array}{l}\text { Traqueo } \\
\text { bronquitis }\end{array}$ & 1 & 0,8 & & & & & & & 2 & 1,7 & 51 & 11,9 & & & & & \\
\hline Flebitis & 1 & 0,8 & & & 4 & 40 & & & 2 & 1,7 & & & 1 & 33,3 & & & \\
\hline Candidemia & & & & & & & & & 1 & 0,9 & & & & & & & \\
\hline Celulitis & & & & & & & & & 4 & 3,4 & & & & & & & \\
\hline Iso Gineco & & & 4 & 26,7 & & & & & & & & & & & & & \\
\hline Sepsis & 2 & 1,5 & & & & & & & 2 & 1,7 & & & & & & & \\
\hline \multicolumn{18}{|l|}{ Meningitis } \\
\hline Iso Cardio & 2 & 1,5 & & & & & & & & & & & & & & & \\
\hline Iso Gastro & 2 & 1,5 & & & & & & & & & & & & & & & \\
\hline Iso Cx Tórax & 1 & 0,8 & & & & & & & & & & & & & & & \\
\hline \multicolumn{18}{|c|}{ Iso Urología } \\
\hline \multirow[t]{3}{*}{ Total General } & 130 & 100 & 15 & 100 & 10 & 100 & 4 & 100 & 116 & 100 & 42 & 100 & 3 & 100 & $36 \quad 1$ & 13 & 100 \\
\hline & \multicolumn{2}{|c|}{$\begin{array}{c}\text { UCI } \\
\text { Adultos }\end{array}$} & \multicolumn{2}{|c|}{$\begin{array}{c}\text { UCI } \\
\text { Gineco }\end{array}$} & \multicolumn{2}{|c|}{$\begin{array}{c}\text { UCI } \\
\text { Pediátrica }\end{array}$} & \multicolumn{2}{|c|}{$\begin{array}{l}\text { Unidad } \\
\text { Básica }\end{array}$} & \multicolumn{2}{|c|}{$\begin{array}{c}\text { Urgencias } \\
\text { Adultos }\end{array}$} & \multicolumn{2}{|c|}{$\begin{array}{l}\text { Urgencias } \\
\text { Pediatría }\end{array}$} & \multicolumn{2}{|c|}{ Urología } & $\begin{array}{r}\mathrm{Tc} \\
\mathrm{Ger}\end{array}$ & $\begin{array}{l}\text { tal } \\
\text { hreal }\end{array}$ & \\
\hline & № & $\%$ & № & $\%$ & № & $\%$ & № & $\%$ & № & $\%$ & № & $\%$ & № & $\%$ & № & $\%$ & \\
\hline Bacteriemia & 102 & 51,5 & 3 & 10,7 & 12 & 75 & & & 1 & 8,3 & 8 & 72,7 & 2 & 18,2 & 2198 & 30,7 & \\
\hline Ivun & 19 & 9,6 & 14 & 50,0 & 4 & 25 & 1 & 100 & 0 & 0 & 1 & 9,1 & 8 & 72,7 & 7128 & 19,8 & \\
\hline $\mathrm{NN}$ & 54 & 27,3 & 2 & 7,1 & & & & & & & & & & & 93 & 14,4 & \\
\hline B.A.C. & 7 & 3,5 & 2 & 7,1 & & & & & 9 & 75 & & & & & 65 & 10,1 & \\
\hline Iso Cx Gral & & & & & & & & & & & & & & & 52 & 8,0 & \\
\hline Iso Ortopedia & & & & & & & & & & & & & & & 29 & 4,5 & \\
\hline Iso Neurocx & & & & & & & & & & & & & & & 19 & 2,9 & \\
\hline $\begin{array}{l}\text { Traqueo } \\
\text { bronquitis }\end{array}$ & 7 & 3,5 & 3 & 10,7 & & & & & & & & & & & 18 & 2,8 & \\
\hline Flebitis & 2 & 1,0 & & & & & & & 1 & 8,3 & & & & & 11 & 1,7 & \\
\hline Candidemia & 2 & 1,0 & 2 & 7,1 & & & & & & & 2 & 18,2 & & & 7 & 1,1 & \\
\hline Celulitis & 2 & 1,0 & & & & & & & 1 & 8,3 & & & & & 7 & 1,1 & \\
\hline Iso Gineco & & & 2 & 7,1 & & & & & & & & & & & 6 & 0,9 & \\
\hline Sepsis & & & & & & & & & & & & & & & 4 & 0,6 & \\
\hline Meningitis & 3 & 1,5 & & & & & & & & & & & & & 3 & 0,5 & \\
\hline Iso Cardio & & & & & & & & & & & & & & & 2 & 0,3 & \\
\hline Iso Gastro & & & & & & & & & & & & & & & 2 & 0,3 & \\
\hline Iso Cx Tórax & & & & & & & & & & & & & & & 1 & 0,2 & \\
\hline Iso Urología & & & & & & & & & & & & & 1 & 9,1 & 1 & 0,2 & \\
\hline Total General & 198 & 100 & 28 & 100 & 16 & 100 & 1 & 100 & 12 & 100 & 11 & 100 & 11 & 100 & 646 & 100 & \\
\hline
\end{tabular}


La Infección de Vías Urinarias (IVU) Nosocomial fue la segunda causa de infecciones intrahospitalarias con un porcentaje de 19,8\% del total de casos que se presentaron principalmente en los servicios de Medicina Interna (46 casos), UCI Adultos (19 casos), Cirugía (17 casos) y UCI Gineco (14 casos). El servicio que mayor volumen de antibiogramas tuvo durante el periodo de estudio fue UCI Adultos en el cual las infecciones intrahospitalarias que en mayor porcentaje se encontraron fueron las Bacteriemias con el 51,5\%, en segundo lugar con el 27,3\% correspondiente a Neumonías Nosocomiales (NN) y en tercer lugar las IVU Nosocomiales con el 9,3\%. El servicio de Cirugía se encuentra en segundo lugar con un reporte de antibiogramas de 130 correspondiente a 20,12\%, de los cuales la principal infección nosocomial asociada fue la del Sitio Operatorio (ISO) con el $40 \%$ de los caso, Bacteriemias con el 18,5\% y las Bacteriemias Asociadas a Catéter (B.A.C.) con el 17,7\% de los reportes.

\section{Sensibilidad y resistencia}

\section{Gram negativos:}

En lo observado en la Tabla 4, de los microorganismos gram negativos registrados como sensibles para Amikacina presentan sensibilidades mayores al 70\% siendo el mayor la E. coli con una sensibilidad del $95,8 \%$ y la menor para Pseudomona con un $72,6 \%$. Con respecto a la resistencia de este antibiótico se observó mayor resistencia para Enterobacter con un 31,3\% y Pseudomona con un 27,4\%.

Con relación a la ampicilina se encontró sensibilidad registrada únicamente para Proteus con un $47,6 \%$ y E.coli con un $16,7 \%$. Se observaron resistencias para ampicilina $>$ al $70 \%$ para Klebsiella, Enterobacter y E.coli.

El antibiótico Ampicilina/Sulbactam presentó una sensibilidad $>$ al $70 \%$ en Proteus seguidas de Acinetobacter con un 39,3\%, Klebsiella con un $33,3 \%$ E.coli con un $26 \%$, los demás gram negativos presentaron sensibilidades < al 10\%. Se encontraron resistencias $\geq 50 \%$ para Acinetobacter, E.coli, Enterobacter y Klebsiella.

Cefalotina presentó sensibilidades < al 15\% en todos los gérmenes gram negativos y la mayor resistencia se observó en Enterobacter con un $47,9 \%$ y E.coli con 35,4\%. Cefepime presentó una sensibilidad $>$ al $50 \%$ para todos los gram negativos a excepción de Acinetobacter que presentó un 37,5\%, las mayores resistencias para Cefepime se observaron en Acinetobacter con un $62,5 \%$ y Klebsiella con un $41,1 \%$.

Con respecto a Cefoxitin se observaron sensibilidades $>70 \%$ para Proteus, Klebsiella y E. coli. Enterobacter presentó una tasa de resistencia del $85,4 \%$, los demás antibióticos presentaron tasas de resistencia inferiores al 25\%. Ceftazidima mostró sensibilidades mayores al 50\% en todos los Gram negativos con excepción de Acinetobacter en la que la sensibilidad fue del $30 \%$ y la resistencia cercana al $70 \%$ seguidos de Klebsiella, E. coli y Pseudomona con valores superiores al $40 \%$.

Ciprofloxacina presentó tasas $\geq 50 \%$ en la totalidad de los gérmenes con excepción de Acinetobacter que tuvo una sensibilidad del $46 \%$. Con respecto a la resistencia se encontró mayor al 45\% para E. coli y Acinetobacter. Pseudomona y Acinetobacter presentaron sensibilidad mayor al 95\% para Colistin sin encontrar casos de resistencia a este antibiótico.

Con Ertapenem se observó sensibilidad mayor al 40\% para Proteus, Klebsiella, y E. coli. Gentamicina se observó sensibilidad mayor al 60\% excepto para Acinetobacter que evidenció una sensibilidad del $41 \%$ y una resistencia del $60 \%$, para los demás gérmenes los valores de resistencia fueron menores al $40 \%$.

Para Meropenem e Imipenem se obtuvieron sensibilidades mayores al 50\% a excepción de Acinetobacter que fue de $42 \%$ y $41 \%$ respectivamente; este mismo microorganismo presentó la 


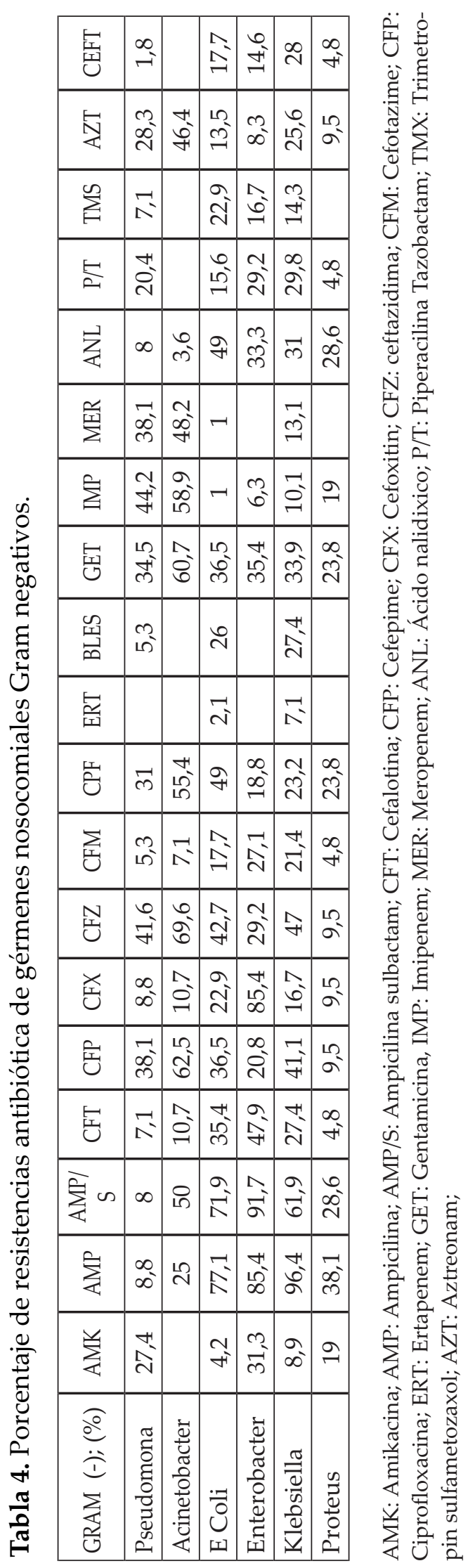

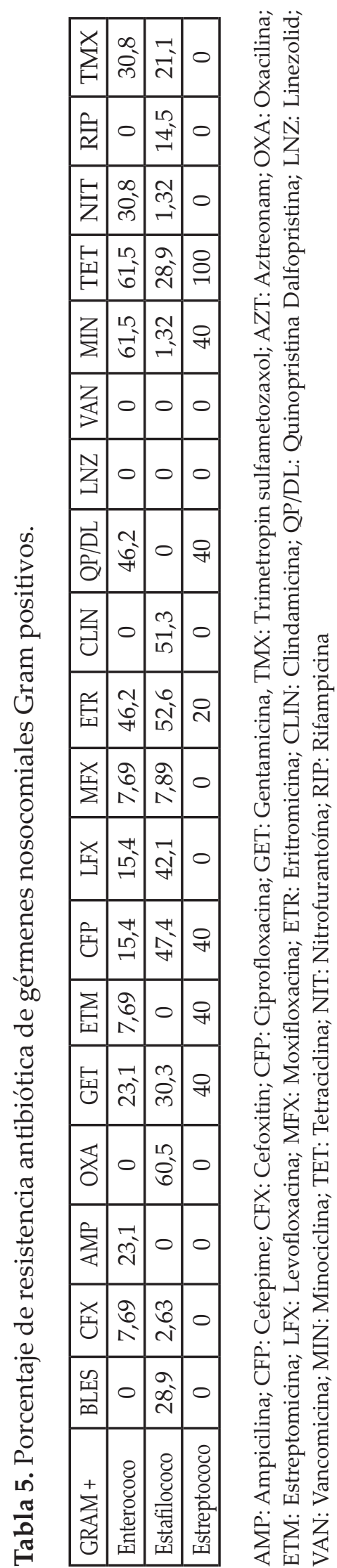


mayor tasa de resistencia para estos dos antibióticos. El Ácido Nalidixico presentó sensibilidad mayor al 60\% para Enterobacter y Klebsiella, y una tasa de resistencia para E.coli del 49\% Piperacilina/Tazobactam mostró el 70\% de sensibilidad para Enterobacter.

Proteus presentó la mayor sensibilidad para Aztreonam siendo esta del 52\% y la mayor resistencia se observó en Acinetobacter en el 46\%; Ceftriaxona evidenció una tasa de sensibilidad mayor del 70\% en Proteus.

\section{Gram positivos:}

En la tabla 5 observamos las tasas de sensibilidad y resistencia para gérmenes gram positivos. En el caso del Enterococo se presentaron sensibilidades mayores al $60 \%$ para los antibióticos de ampicilina, gentamicina, ciprofloxacina, levofloxacina, linezolid, teicoplamina y vancomicina, y se observaron resistencias mayores al $60 \%$ para minociclina y tetraciclina; y blea negativa del $85 \%$.

Para el Estafilococo se observó una sensibilidad mayor al $47 \%$ para los antibióticos Gentamicina, Ciprofloxacina, Levofloxacina, Moxifloxacina, Eritromicina, Clindamicina, Quinupristina/Dalfopristina, Linezolid, Teicoplamina, Vancomicina, Minoclina, Tetraciclina, Nitrofurantoina, Rifampicina y Trimetropin/Sulfametoxazol; y se observó resistencia mayor al 50\% para Oxacilina, Eritromicina y Clindamicina. Estafilococo tuvo una BLEA negativa en el 52,6\% de los casos reportados.

Para el Gram positvo Estafilococo encontramos que los márgenes de resistencia para los principales antibióticos usados en nuestro medio tales como Oxacilina, Clindamicina, Eritromicina y Rifampicina han ido disminuyendo levemente con valores que no superan el 10\% comparado con el estudio anterior realizado en el HUHMP. Del mismo modo vemos que no se han presentado casos de resistencia para los medicamentos de última línea de manejo antibiótico como lo son Linezolid, Teicoplamina y Vancomicina, lo que es un dato alentador para el manejo de este tipo de infecciones.

\section{Concentraciones mínimas inhibitorias de los principales gérmenes}

\section{Klebsiella}

De los antibióticos registrados el de mayor sensibilidad para Klebsiella fue Amikacina con una frecuencia de 153 casos de los cuales $82 \%$ presentaban una MIC < =0,2; seguido a éste el Imipenem con una frecuencia de 151 casos de los cuales $93 \%$ presentaban una MIC < =1, y el meropenem con una frecuencia de 146 casos de los cuales $95 \%$ presentaban una MIC $<=0,25$. La menor sensibilidad antibiótica encontrada corresponde a Cefalotina con una frecuencia de 22 casos de los cuales $95 \%$ presentaban una MIC de $<=2$. De los antibióticos registrados, el de mayor resistencia para Klebsiella fue Ampicilina con una frecuencia de 162 casos de los cuales $90 \%$ presentaban una MIC $>=32$, seguido a este Ampicilina/Sulbactam con una frecuencia de 104 casos de los cuales $89 \%$ presentaban una MIC $>=32$. El menor número de resistencia antibiótica encontrada corresponde a Meropenem, con una frecuencia de 14 casos de los cuales $92,8 \%$ presentaban una MIC de >=16.

\section{Pseudomona}

De los antibióticos registrados, el de mayor sensibilidad para Pseudomona fue Colistin con una frecuencia de 108 casos de los cuales $64 \%$ presentaban una $\mathrm{MIC}=2$, seguido a este Amikacina con una frecuencia de 82 casos de los cuales $51,2 \%$ presentaban una MIC $<=2$, continuando con Ciprofloxacina con una frecuencia de 78 casos de los cuales $65 \%$ presentaban una MIC $<=0,25$, seguido a este Gentamicina con una frecuencia de 73 casos de los cuales $72 \%$ presentaban una MIC <=1. De los antibióticos registrados, el de mayor resistencia para Pseudomona fue Ceftazidima con una frecuencia de 47 casos de los cuales 77,7\% presentaban una MIC >=64, seguido por Cefepime y Meropenem ambos con 
una frecuencia de 43 casos, Cefepime con una MIC > $=64$ y un 62,7\% de casos y Meropenem con una MIC >=16 con $86 \%$ de los casos. La menor resistencia antibiótica encontrada corresponde a Ceftriaxona, con una frecuencia de 2 casos de los cuales $100 \%$ presentaban una MIC de $>=64$.

\section{E. Coli}

Observamos las Concentraciones Mínimas Inhibitorias (MIC) para E. coli. De los antibióticos registrados el de mayor sensibilidad para E. coli fue Meropenem con una frecuencia de 95 casos de los cuales $92 \%$ presentaban una MIC $<=0,25$, seguido por Imipenem con una frecuencia de 93 casos de los cuales $91 \%$ presentaban una MIC $<=1$, continuando com Amikacina con una frecuencia de 92 casos de los cuales 70\% presentaban una MIC $<=2$, seguido por Cefoxitin con una frecuencia de 71 casos de los cuales 59\% presentaban una MIC <=4. La menor sensiblidad antibiótica encontrada corresponde a Colistin con una frecuencia de 2 casos de los cuales $100 \%$ presentaban una MIC de 2.

De los antibióticos registrados, el de mayor resistencia para E. coli fue ampicilina con una frecuencia de 74 casos de los cuales $98,7 \%$ presentaban una MIC >=32, seguido por Ampicilina/ Sulbactam con una frecuencia de 69 casos de los cuales $81 \%$ presentaban una MIC $>=32$, seguido a este tenemos Piperacilina/Tazobactamcon una frecuencia de 47 casos de los cuales $100 \%$ presentaban una MIC $>=128$, seguido a este Ciprofloxacina con una frecuencia de 47 casos de los cuales $91 \%$ presentaban una MIC >=4. La menor resistencia antibiótica encontrada corresponde a Meropenem, con una frecuencia de 1 casos de los cuales $100 \%$ presentaban una MIC de >=16.

\section{Estafilococo}

La mayor sensibilidad registrada para Estafilococo fue de Teicoplanina y Vancomicina ambos con una frecuencia de 76, con una MIC <=0,5 en $39,47 \%$ de los casos para Teicoplanina y una MIC de 1 en un 63,15\% de los casos para vancomicina. Quinupristin/ Dalfopristin les sigue con una frecuencia de 74 de los cuales $86,47 \%$ poseen una $\mathrm{MIC}<=0,25$.

La mayor resistencia antibiótica del Estafilococo se registró para Oxacilina con 46 casos de los cuales el $100 \%$ correspondían a una MIC > $>4$, seguida de Clindamicina con 39 reportes de los cuales $89,74 \%$ correspondían a una MIC >=8.

\section{Acinetobacter}

De los antibióticos registrados los de mayor sensibilidad para Acinectobacter fueron Amikacina con una frecuencia de 50 de los cuales $44 \%$ presentaron una MIC de 16 y Ciprofloxacina con una frecuencia de 26 de los cuales $76,92 \%$ tenían una MIC < =0,25, el Ácido Nalidixico presentó la menor sensibilidad registrando una frecuencia de 4 con una MIC de 4 en 100\% de los casos. La mayor resistencia antibiótica corresponde a la Ceftazidima con una frecuencia de 39 casos y Cefepime con 35 casos ambos presentando MIC $>=64$ en $46,15 \%$ y $82,89 \%$ de los casos totales respectivamente.

\section{Discusión}

Tras el análisis del comportamiento de las infecciones nosocomiales en nuestra institución y comparándola con los reportes tomados de otros estudios podemos inferir inicialmente que la distribución de la población con respecto al género y edad es muy similar a lo encontrado en los estudios ${ }^{(3,4,5)}$, es decir, la mayoría de la población afectada por infecciones de tipo intrahospitalario corresponde a la población masculina dato que está relacionado con la mayor exposición a factores de riesgo que conllevan a patologías o a situaciones que requieren de manejo intrahospitalario ${ }^{(5,6,7,8)}$.

Factores como el volumen de pacientes que maneja cada servicio es importante a tener en cuenta, puesto que unidades como la de Cuidados Intensivos, Cirugía y Medicina Interna, que por su alto número de casos complejos atendidos durante el periodo de estudio, aporta proporcionalmente mayor cantidad de casos asocia- 
dos a infecciones de tipo intrahospitalario ${ }^{(9)}$; del mismo modo al ser estos servicios los de mayor complejidad en nuestro hospital son los que poseen pacientes con mayor número de comorbilidades que precipitan y facilitan la aparición de enfermedades relacionadas al cuidado de la salud tales como varios especialistas que ven un mismo paciente, mayor cantidad de equipos para la sobrevida del paciente, procedimientos más invasivos, patologías asociadas que favorecen la aparición de infecciones, entre otras. Son los servicios de Cirugía y Medicina Interna los que poseen mayor cantidad de camas para alojar pacientes, lo que a su vez, si bien es cierto es bueno, indirectamente condiciona la aparición de infecciones intrahospitalarias.

En nuestro estudio se determinó que el servicio de cirugía general pasó a ocupar el segundo lugar en frecuencia de presentación de infecciones nosocomiales, de las cuales la principal causa fueron las Infecciones de Sitio Operatorio, dato concordante con la publicación titulada "Infección Del Sitio Operatorio En Un Hospital Nivel II" en la cual, las infecciones de sitio operatorio de cirugía general eran las principales en presentación y frecuencia ${ }^{(10)}$.

Con respecto a la distribución de los patógenos generadores de infecciones nosocomiales y comparándolo con uno de los principales estudios de prevalencia de las Infecciones Nosocomiales (EPINE) ${ }^{(11)}$ encontramos que la frecuencia de los agentes causales es diferente, posicionando a Klebsiella como el germen más frecuente en nuestro medio; Pseudomona, E. coli y Estafilococo, al igual que el estudio español $_{11}$, se encuentran dentro de los principales microorganismos asociados a infecciones intrahospitalarias, desplazando a Enterococo que en dicho estudio ocupa el tercer lugar en el nuestro se posiciona en el noveno puesto con el $2 \%$ aproximado de los casos reportados. Los anteriores datos concuerdan al igual con reportes del ámbito nacional, en el estudio realizado en el Hospital San Jerónimo en Montería ${ }^{(12)}$, donde mostraron una diferencia notable con respecto a la distribución de los patógenos asociados a infecciones nosocomiales. Sin embargo, en estudios previos realizados en el HUHMP, encontramos que los patógenos siguen siendo los mismos, pero con frecuencias de presentación diferentes como se expuso en los resultados.

John C. O'Horo, MD1; Dennis G. Maki y cols. ${ }^{13}$ en un metaanalisis encontró la bacteremia asociada a catéter como causa importante de infecciones nosocomiales. Nosotros la encontramos en un $10 \%$ siendo la unidad de cuidado intensivo el lugar de hospitalización más relacionado. Ivana Milosevi y Milos Kora et al. ${ }^{(14)}$ en su estudio sobre infecciones nosocomiales en un hospital de Serbia mostraron que la infección del tracto urinario nosocomial se relacionó en un $41 \%$, la neumonía y la sepsis de tejidos blandos en un $26 \%$ y $21 \%$, respectivamente. Nosotros encontramos una proporción de 19,8\%, 14,4\% y $1,1 \%$ respectivamente. Estos datos discrepantes pueden estar explicados por el número de pacientes tomados por el estudio de referencia que fue solo de 33 pacientes con cultivos; si embargo el orden de frecuencia de las infecciones nosocomiales guarda relación.

Alvarez Carlos, Cortez Jorge et al., ${ }^{(15)}$ en un estudio titulado "Resistencia antimicrobiana en unidades de cuidado intensivo en Bogotá", encontraron que el Estafilococcus aureus, la E coli, la Pseudomonas aeuriginosa y la Klebsiella pneumoniae son los gérmenes intrahospitalarios más relacionados, siendo este hallazgo parecido a lo encontrado en nuestra investigación. Las tasas de resistencia para estafilococus a oxacilina fueron del 61 al $63 \%$, en nuestro estudio este dato fue similar $(60 \%)$. La resistencia de la $E$ coli a las cefalosporinas de primera generación fue del $10 \%$ y del $20 \%$ a ciprofloxacina, nosotros encontramos una resistencia del $35 \%$ a cefalotina y del $49 \%$ a ciprofloxacina; hallazgos que estan muy por encima de lo comparado con el estudio. Para Klebsiella pneumoniae la resistencia a cefalosporinas de tercera generación fue superior al $30 \%$, siendo para nosotros muy similar (28\%). 
Chavez Monica, Salazar Martha y colaboradores $^{(16)}$ en su estudio títulado "Bacterias resistentes a los antibióticos en infecciones nosocomiales de un hospital de Cali (Colombia)", tomaron 1899 cultivos encontrando que la Escherichia coli, la Klebsiella pneumoniae y el Proteus mirabilis, son los que más se manifestaron, con resistencia variable a betalactámicos exceptuando los carbapenémicos. La Pseudomonas aeruginosa presentó resistencia a los betalactámicos, gentamicina en un 16,8\%, quinolonas en un $23,9 \%$. El Proteus mirabilis, Enterobacter aerogenes, Citrobacter freundii y Klebsiella pneumoniae presentaron resistencia a cefoxitina, cefalosporinas de tercera generación, inhibidores de betalactamasa, y sensibilidad a cefepime. Nuestro estudio muestra hallazgos similares, tanto en porcentaje de frecuencia de los gérmenes como porcentaje de resistencia antimicrobiana.

Del mismo modo, en este estudio logramos determinar factores importantes como la concentración mínima inhibitoria (MIC) en el manejo farmacológico de las infecciones nosocomiales, dato que hasta el momento no había sido tenido en cuenta para un tamizaje epidemiológico; este dato permite valorar con qué concentraciones del medicamento se logra inactivar al $90 \%$ de la cepa en cultivo determinando así las dosis farmacológicas y el tipo de medicamento idóneo según la infección nosocomial presentada y su organismo desencadenante. Una de las posibles causas del aumento de la resistencia microbiológica presentada, obedece a que no en todas las unidades de atención en salud se tienen en cuenta las Concentraciones Mínimas Inhibitorias y existen pocos estudios en la literatura nacional que optimicen el manejo farmacológico de infecciones en el ámbito intrahospitalario teniendo en cuenta las MIC dependiendo de la sensibilidad y resistencia según el perfil epidemiológico de la institución ${ }^{(17)}$.

Dentro de las limitaciones del estudio encontramos que los antibiogramas para gram negativos y gram positivos solo mostraban los fármacos activos contra este grupo de bacterias sin lograr evaluar las resistencias naturales.

\section{Conflictos de intereses:}

Los autores declaran que en la realización del estudio no se presentaron conflictos de intereses.

\section{Conclusiones}

De la totalidad de las infecciones reportadas en el periodo de estudio encontramos como principales causas de infecciones nosocomiales en el Hospital Universitario Hernando Moncaleano Perdomo, en orden de frecuencia descendente, a Klebsiella, Pseudomona, E. coli, Estafilococo y Acinetobacter, como patógenos desencadenantes asociados. Las principales patologías asociadas a infecciones nosocomiales fueron Bacteriemia, Infección de Vías Urinarias Nosocomial, Neumonía Nosocomial, Bacteriemia Asociada a Catéter e Infecciones de Sitio Operatorio de Cirugía General

El actual manejo farmacológico para infecciones nosocomiales que se está llevando a cabo tras la adopción de las Guías de Manejo Antibiótico del HUHMP ha contribuido de manera significativa para la disminución de las tasas de resistencia y un aumento moderado en la sensibilidad antibiótica para las principales infecciones nosocomiales. Sin embargo patógenos como Klebsiella y E. coli presentaron aumento en las tasas de resistencia para algunos antibióticos específicos superiores al 10\% lo que podría estar asociado al uso indiscriminado de algunos medicamentos en las terapias empíricas iniciales.

Pseudomona presentó un aumento en la tasa de resistencia para medicamentos de amplio espectro tales como los carbapenémicos de aproximadamente el 20\%. Los gérmenes Gram positivos relacionados con infecciones nosocomiales se encuentra en mayor proporción al Estafilococo con más del $80 \%$ de los casos para este tipo de gérmenes; en menor frecuencia se encuentra Estreptococo y Enterococo; de los antibióticos utilizados para infección nosocomial por estafi- 
lococo se ha presentado una disminución de las tasas de resistencia y ningún caso de resistencia para medicamentos de amplio espectro como Linezolid, Vancomicina y Teicoplamina. De las infecciones nosocomiales producidas por hongos la Cándida representa el 100\% de los casos aislados

\section{Bibliografía}

1. J.J. SANCHO INSENSER. Epidemiología y etiología quirúrgica de las complicaciones infecciosas postoperatorias. En: Álvarez Francisco, Complicaciones infecciosas en el postoperatorio de cirugía abdominal. Universidad autónoma de Barcelona, Hospital Universitario del mar. Ediciones Ergon; Barcelona. 2000, p: 3-20.

2. ARCHIBALD L, PHILLIPS L, MONNET D, MCGOWAN J, TENOVER F, GAYNES R. Antimicrobial Resistance In Isolates From Inpatients And Outpatients In The United States: Increasing Importance Of The Intensive Care Unit. Clin Infect Dis. 1997; 24: 211-5.

3. BIRGAND, G, RADU C. Does A GentamicinImpregnated Collagen Sponge Reduce Sterna Wound Infections In High-Risk Cardiac Surgery Patients? Interactive CardioVascular and Thoracic Surgery. 2012; 0: 1-8.

4. SOFTAH A, BEDARD A, HENDRY P, MASTERS R, GOLDSTEIN W, BRAIS M, KEON W. Wound Infection In Cardiac Surgery. Annals of Saudi Medicine, 2012; (22): 1 -2.

5. TEENA C, JING J Z, ALANGADEN G. Preventing surgical site infections after bariatric surgery: value of perioperative antibiotic regimens. Rev Pharmacoecon Outcomes Res. 2010 June; 10(3): 317-328.

6. WEINSTEIN, R. Epidemiology and Control Of Nosocomial Infections In Adult. Intensive Care Units. American Journal Medicine. 1991; 91:179-84.

7. CRAVEN DE; HJALMARSON KI. VentilatorAssociated Tracheobronchitis and Pneumonia: Thinking Outside the Box. Clinical Infectious Diseases 2010; 51(S1): 59-66.
8. WEINSTEIN, RA. Nosocomial Infection Update. Emerging Infectious Diseases. Chicago, Illinois. Vol. 4, (3): 416-420.

9. CUÉTARA M, ALAMBRA A, PALACIO A. Diagnóstico Microbiológico Tradicional de la Candidiasis Invasora en el enfermo crítico no neutropénico. Revista Iberoamericana de Micologia. 2006; (23): 4-7.

10. ÍNIIGO J, BERMEJO B, ORONOZ B. Infección de sitio quirúrgico en un servicio de cirugía general. Análisis de cinco años y valoración del índice National Nosocomial Infection Surveillance (NNIS). Revista Cirugía Española. 2006; 79 (4):224-30.

11. GARCIA MC, CHAMORRO J. Prevalencia de la infección nosocomial en Navarra. Resultados del estudio EPINE. Anales Sistema Sanitario Navarra. 2007, Vol. 30, № 1,p: 89-99.

12. PERES D, MATTIR S. Alta resistencia de los Microorganismos Nosocomiales en el Hospital San Jerónimo de Montería. Centro de investigación en infectología y microbiología. Montería (Cordoba). 2003.

13. JOHN C. O'HORO; DENNIS G. MAKI. Arterial catheters as a source of bloodstream infection: a systematic review and meta-analysis. Critical care medicine. 2014. January 9

14. IVANA MILOSEVI, MILOS KORA. Nosocomial infections in the Intensive Care Unit, University Hospital for Infectious and Tropical Diseases, Belgrade, Serbia. Vojnosanit Pregl 2014; 71(2): 131-136.

15. ÁLVAREZ C, CORTEZ J, Resistencia Antimicrobiana en Unidades de Cuidado Intensivo de Bogotá, Colombia (2001-2003). Revista de salud pública, 8 (1): 2006: 86-101.

16. CHAVEZ M, SALAZAR M. Bacterias resistentes a los antibióticos en infecciones nosocomiales de un hospital en Colombia. Enfermedades infecciosas y microbiología. 2012, 33 (1): 19-25.

17. GÓMEZ C, OSORIO J, SANTOFIMIO D. Guías De Manejo Antibiótico Primera Edición. Hospital Universitario Hernando Moncaleano Perdomo. Neiva (Huila) 2011. 